\title{
Incidence of Rhinosporidiosis in Jharkhand
}

\author{
Dr. Ranbir Kumar Pandey ${ }^{1}$,Dr. Amit Kumar ${ }^{2}$,Dr. Dheeraj Kumar ${ }^{3}$ \\ ${ }^{1}$ Associate Professor, Department Of Otorhinolaryngology, Rajendra Institute Of Medical Sciences, Ranchi. \\ ${ }^{2}$ Senior Resident, Department Of Otorhinolaryngology, Rajendra Institute Of Medical Sciences, Ranchi. \\ ${ }^{3}$ Junior Resident, Department Of Otorhinolaryngology, Rajendra Institute Of Medical Sciences, Ranchi.
}

\begin{abstract}
:
Introduction: Rhinosporidiosis is a disease caused by Rhinosporidium seeberi which primarily affects the mucosa of the nose, conjunctiva and urethra. Rhinosporidiosis is endemic disease which has affected various parts of the world, most notably India and Sri Lanka. Its manifestation is a polypoid mass growing inside the affected cavity and the only treatment is surgical excision. Rhinosporidiosis is a condition which both clinicians and microbiologists should keep in mind when managing patients with nasal masses even those from non endemic areas. It is critical in such cases to follow the clinical course to ensure against recurrence of the disease. This study describes the clinical features, diagnosis, and treatment of rhinosporidiosis of the nose and nasopharynx in Jharkhand.

Material and Method: The retrospective study population consisted of patients presented to ENT department at Rajendra Institute of Medical Sciences, Ranchi with nasal mass associated with nasal bleeding. Patients was examined systematically giving due importance to local examination. After clinical diagnosis, Surgical treatment given and further investigations were done for the confirmation of diagnosis. Treatment instituted appropriately and follow up done.

Results: A male gender and rural residential status has high incidence of rhinosporidiosis. Bathing in rivers and ponds were found to be strongly associated with rhinosporidiosis. Almost all patients were found to be of low socioeconomic status. Nasal obstruction and epistaxis were the most common presenting complaints.

Conclusion: Rhinosporidiosis is common in male gender, young and middle aged adults, rural residential status, a history of bathing in ponds and rivers and a low socioeconomic status. Post-surgical recurrence of rhinosporidial masses can be avoided with careful and complete clearance of the mass and cauterization of the base. Appropriate and consistent public health education helps to reduce the incidence of rhinosporidiosis in endemic communities.
\end{abstract}

Keywords: Rhinosporidiosis, Nasal mass, Surgical excision, Nasal obstruction, Dapsone Therapy.

\section{Introduction}

Rhinosporidiosis has been known for over 100 years since its first description by Guillermo Seeber in Buenos Aires in Argentina. It occurs universally, although it is endemic in south Asia, notably southern India and in Sri Lanka. Increased migration to the west of persons who acquired rhinosporidiosis in their native Asian countries has resulted in the increasing occurrence of this disease in the West ${ }^{1}$.

Rhinosporidiosis is a chronic, granulomatous disease process which is characterized by reddish polypoidal masses which are hyperplastic and friable and may be pedunculated or sessile. Rhinosporidium seeberi has been found to have an affinity for the mucous membranes of the nasal cavity and nasopharynx, mainly affecting the anterior nasal septum and vestibule. Extranasal involvement is rare, particularly that involving the lower aerodigestive tract including the tracheobronchial tree. Various other sites have been reported in literature and these include conjunctiva, lips, uvula, palate, trachea, larynx, vagina, penis and bone ${ }^{2,3}$. The presumed mode of infection from the natural aquatic habitat of Rhinosporidium seeberi is through the traumatized epithelium ('transepithelial infection') most commonly in nasal sites ${ }^{4}$.

Guillermo Seeber proposed the infective aetiology for this disease to be a fungus, which was later isolated by Ashworth in 1923, who in turn described the life cycle of the organism and established the nomenclature Rhinosporidium seeberi ${ }^{5}$. The infectious agent forms round and thick-walled sporangia in the submucosa of the affected site, varying from 10-200 mm in size, which are visible as white dots in the mucosa containing smaller 'daughter cells' (called 'sporangiospores'). It can be visualized with fungal stains such as Gomori methenamine silver (GMS) and periodic acid-Schiff (PAS), as well as with standard haematoxylin and eosin (H\&E) staining.

The disease is more common in younger age groups as has been observed by various authors. An uncommon pathogen, typically restricted to tropical areas and seems to occur more in the younger age group, more so in men, as this group is frequently occupationally active (agriculturists, sand workers, divers etc). Less outdoor activity and less chance of contact with animals could explain fewer incidences among women ${ }^{6}$. 
Spontaneous regression of rhinosporidial growths has been noted in animals and in humans but is rare. Therefore, medical and/or surgical intervention is necessary. Wide local surgical excision with electrocoagulation of the base of the lesions is the treatment of choice to reduce the risk of recurrence. The only drug appearing to have clinical promise is Dapsone ${ }^{7}$. It arrests the maturation of sporangia and promotes fibrosis in the stroma, when used as an adjunct to surgery ${ }^{4}$.

\section{Materials And Methodology}

This was a retrospective study involving 45 patients of histologically proven rhinosporidiosis treated in the Department of ENT at Rajendra Institute of Medical Sciences, Ranchi, over a period of 1 year. All patients had undergone a detailed history taking and clinical examination which were retrieved from hospital case records and personal interviews. Detailed history included duration of symptoms, personal habits, area of residence, bathing habits and work profile of the patient. Diagnosis was made on the basis of detailed history, clinical examination and investigations including Plain X-ray Nose and Paranasal Sinuses, CT Paranasal Sinuses (coronal and axial views), Diagnostic nasal endoscopy and histopathological examination. All patients were subjected to routine haematological investigations including blood grouping for operative fitness and excision of the mass and electrocautery of the base of lesion under general anaesthesia. Following excision, the entire nasal cavity and nasopharynx were examined for any lesions left behind. All excised specimens were sent for histopathological examination and confirmation of the diagnosis. All patients had been followed up for a period of 12 months and were subjected to clinical examination and diagnostic nasal endoscopy at regular intervals at 2 weeks, 3 months, 6 months and at the end of one year.

\section{Results}

Statistical Analysis: The data was analyzed by using SPSS 20 software. The data is presented in percentages, rates and ratios. Chi square test was used to find the association between attributes.

Out of 100 cases which were clinically diagnosed as cervical tubercular lymphadenitis 80 cases were confirmed as tubercular lymphadenitis, 15 cases turned out as reactive lymphadenitis, 3 cases reported as Lymphoma and 2 cases were found to have Metastatic secondary's. Confirmed tubercular lymphadenitis cases were analyzed further.

'Age-sex distribution: Of these 45 cases, 26 (57.8\%) were male and 19 (42.2\%) were females. The male to female ratio was found to be 1.4:1. Most cases were found to fall within the 11-30 years age group, with the maximum in the second and third decades of life, but no statistically significant relationship could be found between age and incidence of rhinosporidiosis.

Table - I: Age-sex distribution

\begin{tabular}{|l|l|l|l|}
\hline Age & Male & Female & Total \\
\hline $0-10$ & $03(6.7 \%)$ & $01(2 \%)$ & $04(8.8 \%)$ \\
\hline $11-20$ & $10(22.2 \%)$ & $09(20 \%)$ & $19(42.2 \%)$ \\
\hline $21-30$ & $08(17.8 \%)$ & $04(8.9 \%)$ & $12(26.7 \%)$ \\
\hline $31-40$ & $03(6.7 \%)$ & $03(6.7 \%)$ & $06(13.3 \%)$ \\
\hline $41-50$ & $01(2.2 \%)$ & $01(2.2 \%)$ & $02(4.4 \%)$ \\
\hline$>50$ & $01(2.2 \%)$ & $01(2.2 \%)$ & $02(4.4 \%)$ \\
\hline Total & $26(57.8 \%)$ & $19(42.2 \%)$ & $45(100 \%)$ \\
\hline
\end{tabular}

Etiological factor: A multiple etiological factors was conducted including occupation, residential status, socioeconomic status and bathing history. The patients in this study were mainly farmers, housewives or students, with a few daily wage laborers.

The majority of cases lived in rural areas (84.4\%), while the remaining lived in urban (15.6\%) areas. A significant relationship was found between rural residential status and incidence of rhinosporidiosis. Almost all patients gave a history of bathing in ponds $(71 \%)$ or rivers $(26.7 \%)$. Almost all patients were found to be of low socioeconomic status $(77.8 \%)$ as compared to $22.2 \%$ of patients who were of middle socioeconomic status.

Table - II: Etiological factor

\begin{tabular}{|l|l|l|}
\hline Etiology & \multicolumn{2}{|l|}{ Number of cases } \\
\hline \multirow{3}{*}{ Occupation } & Farmer & $17(37.8 \%)$ \\
\cline { 2 - 3 } & Daily wage labourer & $02(4.4 \%)$ \\
\cline { 2 - 3 } & Student & $14(31 \%)$ \\
\cline { 2 - 3 } & Housewife & $12(26.7 \%)$ \\
\hline \multirow{2}{*}{ Residential status } & Rural & $38(84.4 \%)$ \\
\cline { 2 - 3 } & Urban & $07(15.6 \%)$ \\
\hline Bathing history & Ponds & $32(71 \%)$ \\
\cline { 2 - 3 } & Rivers & $12(26.7 \%)$ \\
\hline
\end{tabular}




\begin{tabular}{|l|l|l|}
\hline & Tap water & $01(2.2 \%)$ \\
\hline \multirow{2}{*}{$\begin{array}{l}\text { Socioeconomic } \\
\text { status }\end{array}$} & Low & $35(77.8 \%)$ \\
\cline { 2 - 3 } & Middle & $10(22.2 \%)$ \\
\cline { 2 - 3 } & High & 0 \\
\hline
\end{tabular}

Clinical features: The symptoms with which the patients presented to the outpatient department included nasal obstruction, epistaxis, nasal discharge and complaints of nasal mass. Of these, nasal mass (84.4\%), nasal obstruction (93\%) and epistaxis (66.7\%) were the most common presenting complaints among patients with rhinosporidiosis

Table - III: Clinical features

\begin{tabular}{|l|l|l|}
\hline Clinical features & Number of cases $(\mathrm{N}=80)$ & Percentage \\
\hline Nasal mass & 38 & $84.4 \%$ \\
\hline Nasal obstruction & 42 & $93 \%$ \\
\hline Epistaxis & 30 & $66.7 \%$ \\
\hline Nasal discharge & 15 & $33.3 \%$ \\
\hline
\end{tabular}

Postoperatively all patients were followed up up to 12 months. Of the 45 patients in this study, 4 patients were found to have recurrence $(8.9 \%)$. This low recurrence rate may be explained by careful and complete excision of the rhinosporidial masses with basal cautery, careful counseling of patients following treatment regarding susceptible habits in rhinosporidiosis and preventive measures and regular follow-up with endoscopic examination at each visit.

\section{Discussion}

This study showed a higher prevalence of rhinosporidiosis among males. This finding agrees with those of other authors, as Arsecularatne et $\mathrm{al}^{4}$., and Grover et $\mathrm{al}^{8}$., also showed a higher male preponderance in their respective studies. Probably since females have a lesser chance of animal contact and less frequent pond baths, this may account for the lower female prevalence. Some authors also opined that the effect of estrogen in females may provide some protection from the disease ${ }^{7}$. This study found that the age group most commonly affected was the $11-30$ years age group. Studies by Kutty et $\mathrm{al}^{9}$., and Guru RK and Pradhan $\mathrm{DK}^{10}$., found the maximum number of cases in the age-group $21-30$ years. Thus, it can be concluded that young and middle aged adults are the most commonly affected.

This study shows majority of the patients belongs to low socioeconomic status. Arsecularatne ${ }^{4}$, Guru and $\operatorname{Pradhan}^{10}$ and Jain and Sahal ${ }^{11}$ also found in their respective studies a correlation between low socioeconomic status and occurrence of rhinosporidiosis. The probable reason for this may be the poorer standards of hygiene among people of low socioeconomic status. Patients in this study most commonly presented with complaints of nasal obstruction, followed by nasal mass, epistaxis and nasal discharge.

All cases of nasal and nasopharyngeal rhinosporidiosis were treated by wide excision and cauterization of the base by diathermy and all of them were treated subsequently with Dapsone.

\section{Conclusion}

Rhinosporidiosis is common in male gender, young and middle aged adults, rural residential status, a history of bathing in ponds and rivers and a low socioeconomic status. Post-surgical recurrence of rhinosporidial masses can be avoided with careful and complete clearance of the mass and cauterization of the base. This study also shows that a strong system of public health education may help to reduce the incidence of this disease.

\section{References}

[1]. Morelli L, Polce M, Piscioli F, et al. Human nasal rhinosporidiosis: an Italian case report, Diagn Pathol , 2006, vol. 1 pg. 25

[2]. Franca GV Jr, Gomes CC, Sakano E, Altermani Am, Shimizu Lt. Nasal rhinosporidiosis in children. J Pedatr (Rio J) 1994;70:299301 .

[3]. Makannavar JH, Chavan SS. Rhinosporidiosis: a clinicopathological study of 34 cases. Indian J Pathol Microbiol. 2001;44:17-21.

[4]. Arseculeratne SN. Recent advances in rhinosporidiosis and Rhinosporidium seeberi, Indian J Med Microbiol, 2002, vol. 20 (pg. 119 - 131).

[5]. Karunaratne WA. Rhinosporidiosis in man. London: Athlone Press; 1964. pp. 14-18.

[6]. Chao SS, Loh KS. Rhinosporidiosis: an unusual cause of nasal masses gains prominence, Singapore Med J, 2004, vol. 45 (pg. 224226).

[7]. Venkateswaran S, Date A, Job A, Mathan M. Light and electron microscopic findings in rhinosporidiosis after Dapsone therapy, Trop Med Int Health, 1997, vol. 2 (pg. 1128-1132).

[8]. Grover S. Rhinosporidiosis. J Med Med Ass. 1975;64(9):93-95.

[9]. Kutty MK, Shridharan T, Mathew KT. Some observation on rhinosporidiosis. Am J Med Sci. 1963;246:695-701.

[10]. Guru RK, Pradhan DK. Rhinosporidiosis with special reference to extra nasal presentation. J Evolution Med Dent Sci. 2014;22(3):6189-99.

[11]. Jain MR, Sahal R. Rhinosporidiosis of lacrimal sac. Ind J Opth. 1974;22:29-31. 